This is the accepted manuscript made available via CHORUS. The article has been published as:

Critical temperature and correlation length of an elastic interaction model for spin-crossover materials

Taro Nakada, Takashi Mori, Seiji Miyashita, Masamichi Nishino, Synge Todo, William Nicolazzi, and Per Arne Rikvold

Phys. Rev. B 85, 054408 - Published 7 February 2012

DOI: 10.1103/PhysRevB.85.054408 


\title{
Critical temperature and correlation length of an elastic interaction model for spin-crossover materials
}

\author{
Taro Nakada ${ }^{1}$, Takashi Mori $^{1}$, Seiji Miyashita ${ }^{1,2}$, Masamichi Nishino ${ }^{3}$, \\ Synge Todo ${ }^{2,4}$, William Nicolazzi ${ }^{4,5}$, and Per Arne Rikvold ${ }^{6}$ \\ ${ }^{1}$ Department of Physics, Graduate School of Science, \\ The University of Tokyo, 7-3-1 Hongo, Bunkyo-Ku, Tokyo 113-8656, Japan. \\ ${ }^{2}$ CREST, JST, 4-1-8 Honcho Kawaguchi, Saitama 332-0012, Japan. \\ ${ }^{3}$ National Institute for Materials Science, Tsukuba, Ibaraki 305-0047, Japan. \\ ${ }^{4}$ Department of Applied Physics, University of Tokyo, Tokyo 113-8656, \\ Japan. ${ }^{5}$ CNRS,Laboratoire de Chimie de Coordination (LCC/CNRS/UPR8241) 205, \\ route de Narbonne, 3107 ry Toulouse cedex4, France. \\ ${ }^{6}$ Department of Physics, Florida State University, Tallahassee, Florida 32306-4350, USA.
}

(Dated: January 23, 2012)

\begin{abstract}
It has previously been pointed out that the coexistence of infinite-range and short-range interactions causes a system to have a phase transition of the mean-field universality class, in which the cluster size is finite even at the critical point. In the present paper, we study this property in a model of bistable molecules, whose size changes depending on the bistable states. The molecules can move in space, interacting via an elastic interaction. It is known that due to the different sizes, an effective long-range interaction between the spins appears, and thus this model has a mean-field type of phase transition. It is found that the scaling properties of the shift of the critical temperature from the pure short-range limit in the model with infinite-range and short-range interactions hold also in the present model, regarding the ratio of the size of the two states as a control parameter for the strength of the long-range interaction. By studying the structure factor, it is shown that the dependence of the cluster size at the critical temperature also shows the same scaling properties as a previously studied model with both infinite-range and short-range interactions. We therefore conclude that these scaling relations hold universally in hybrid models with both short-range and weak long-range interactions.
\end{abstract}

PACS numbers:

\section{INTRODUCTION}

The divergence of the correlation length at the critical point is considered to be one of the most important properties of second-order phase transitions. However, it is also known that in phase transitions of infinite-range interacting systems that belong to the mean-field universality class, the correlation length does not diverge, and the spatial configuration is uniform with no domain structures or clustering. In a previous work, we studied a hybrid model with both short-range and weak long-range interactions. ${ }^{1}$ The Hamiltonian of that model is given by

$$
\mathcal{H}=(1-\alpha) \mathcal{H}_{\mathrm{IS}}+\alpha \mathcal{H}_{\mathrm{MF}}, \quad(0 \leq \alpha \leq 1)
$$

with

$$
\mathcal{H}_{\mathrm{IS}}=-J \sum_{\langle i, j\rangle} \sigma_{i} \sigma_{j}
$$

and

$$
\mathcal{H}_{\mathrm{MF}}=-\frac{4 J}{2 N} \sum_{i, j} \sigma_{i} \sigma_{j}=-\frac{2 J}{N} \sum_{i, j} \sigma_{i} \sigma_{j} .
$$

We defined the model on the square lattice with periodic boundary conditions. Here, the strength of the infinite-range interaction is controlled by varying $\alpha$. When $\alpha=0$, the system is equivalent to the pure short-range Ising model, and the system with $\alpha=1$ is equivalent to the pure infinite-range interaction model. The critical temperature in the previous hybrid model (1) shows a crossover from that of the pure short-range Ising model to that of the infiniterange interaction model. It should be noted that even for infinitesimally small $\alpha$, the phase transition belongs to the mean-field universality class, and at the critical point, the spin configuration is uniform with no large-scale clustering. A scaling formula for the $\alpha$-dependence of the critical temperature $T_{\mathrm{c}}$ is found, such that

$$
\frac{T_{\mathrm{c}}(\alpha)-T_{\mathrm{c}}^{\mathrm{IS}}}{T_{\mathrm{c}}^{\mathrm{MF}}-T_{\mathrm{c}}^{\mathrm{IS}}} \simeq 1.773517 \alpha^{\frac{1}{\gamma}}=1.773517 \alpha^{\frac{4}{7}} .
$$


The correlation length $\xi_{\mathrm{c}}$ at $T_{\mathrm{c}}$ is also found as a function of $\alpha$,

$$
\xi_{\mathrm{c}}(\alpha, L)=L f\left(L \alpha^{\frac{\nu}{\gamma}}\right)=L f\left(L \alpha^{\frac{4}{7}}\right)
$$

for small $\alpha$ and large $L$. The function $f(x)$ is a scaling function which asymptotically approaches $1 / x$ for large $x$.

Although the long-range interaction in this model is rather artificial, recently it has been pointed out that spincrossover materials and related materials show a similar kind of long-range correlation. ${ }^{2}$ Spin-crossover materials are molecular crystals, in which the molecules can exist in two different states: the high-spin (HS) state and the low-spin (LS) state. The HS state is preferable at high temperatures because of its high degeneracy, while the LS state is preferable at low temperatures because of its low enthalpy. In addition to temperature, pressure changes and light exposure also often induce a phase transition in spin-crossover materials. Spin-crossover and related materials are used in many applications, because of their inherent bistability that leads to changes in optical and magnetic properties, etc. ${ }^{3}$ Phase transitions in spin-crossover and related materials have been studied extensively in chemistry ${ }^{4-10}$ and recently also in physics. ${ }^{11-16}$ For a wide variety of applications, it is of great interest to study the ordering process in spin-crossover materials.

This type of materials are also regarded as fundamental models for inter-molecular short-range and elastic interactions due to the lattice distortion, and an elastic interaction model has been proposed. ${ }^{2,11}$ An important characteristic of this model is an effective long-range interaction due to the lattice distortion caused by the size difference between the HS (large) and LS (small) molecules. Even at the critical point, there exists no large-scale domain structures. However, in the elastic interaction model, there does not exist inter-molecular short-range interaction, so the model does not show any crossover from an effective short-range interacting system to an effective long-range interacting system. Therefore, developing quantitative hybrid models with both short-range and the elastic interactions for the critical behavior of such materials is very important.

In this paper, we focus on a system with both elastic interactions and short-range Ising interactions. We perform Monte Carlo (MC) simulations to study the properties of this model at the critical point. The rest of the paper is organized as follows. In Sec. II, we introduce a new model with both elastic and short-range Ising interactions, and we propose a relation between this model and the model studied previously. ${ }^{1}$ We find that the scaling formulae for the critical temperature and correlation length obtained in the previous paper ${ }^{1}$ apply to this model, as well. In Sec. III, we briefly review the MC algorithms and give the result of MC simulations for the critical temperature, confirming the relation. In Sec. IV, we similarly confirm the scaling form of the correlation length at the critical point. In Sec. $\mathrm{V}$, we summarize our results, and in appendix A we discuss in detail how we calculate the correlation length at the critical point.

\section{MODEL}

In this paper, we adopt the following model with both lattice distortion and inter-molecular short-range interactions on the square lattice with periodic boundary conditions. From now on, $L$ denotes the number of molecules along an edge of the lattice, so the total number of sites is $N=L^{2}$. The Hamiltonian is

$$
\mathcal{H}=\mathcal{H}_{\mathrm{IS}}+\mathcal{H}_{\mathrm{nn}}+\mathcal{H}_{\mathrm{nnn}}+\mathcal{H}_{\mathrm{eff}}
$$

with

$$
\left\{\begin{array}{l}
\mathcal{H}_{\mathrm{IS}}=-J \sum_{\langle i, j\rangle} \sigma_{i} \sigma_{j} \\
\mathcal{H}_{\mathrm{nn}}=\frac{k_{1}}{2} \sum_{\langle i, j\rangle}\left[\left|\boldsymbol{r}_{i}-\boldsymbol{r}_{j}\right|-\left(R_{i}\left(\sigma_{i}\right)+R_{j}\left(\sigma_{j}\right)\right)\right]^{2} \\
\mathcal{H}_{\mathrm{nnn}}=\frac{k_{2}}{2} \sum_{\langle\langle l, m\rangle\rangle}\left[\left|\boldsymbol{r}_{l}-\boldsymbol{r}_{m}\right|-\sqrt{2}\left(R_{l}\left(\sigma_{l}\right)+R_{m}\left(\sigma_{m}\right)\right)\right]^{2} \\
\mathcal{H}_{\mathrm{eff}}=\left(D-\frac{k_{\mathrm{B}} T}{2} \log g\right) \sum_{i}^{N} \sigma_{i}
\end{array}\right.
$$

where $\boldsymbol{r}_{i}$ represents the continuous coordinate of the molecule $i$, and $R_{i}\left(\sigma_{i}\right)$ is the radius of the molecule $i$ which depends on the molecular state $\sigma_{i}$. Here $\sigma_{i}=+1$ and -1 represents HS and LS state, respectively. Hereafter, we simply call $\sigma_{i}$ the spin state. $\mathcal{H}_{\mathrm{IS}}$ is the short-range pure Ising model. $\mathcal{H}_{\mathrm{nn}}$ and $\mathcal{H}_{\mathrm{nnn}}$ denote the elastic interaction Hamiltonians of nearest-neighbor $\langle i, j\rangle$ and next-nearest-neighbor $\langle\langle l, m\rangle\rangle$ pairs, respectively, and $k_{1}$ and $k_{2}$ are the corresponding spring constants. The next-nearest-neighbor interaction is introduced to maintain the shape of the lattice, and the strength of $k_{2}$ is not important as long as the global shape of the square lattice is kept. Here we take $k_{2}=k_{1} / 10$. We define the pure elastic interaction model as

$$
\mathcal{H}_{\text {Elastic }} \equiv \mathcal{H}_{\mathrm{nn}}+\mathcal{H}_{\mathrm{nnn}}
$$


The molecular radius is determined by the local spin state $: R_{\mathrm{H}}$ for the HS state (large, $\left.\sigma_{i}=+1\right)$ and $R_{\mathrm{L}}$ for the LS state (small, $\sigma_{i}=-1$ ). When $\left|\boldsymbol{r}_{i}-\boldsymbol{r}_{j}\right|$ is equal to the sum of the radii $R_{i}\left(\sigma_{i}\right)+R_{j}\left(\sigma_{j}\right)$, the corresponding contribution to the elastic energy has its minimum. In $\mathcal{H}_{\text {eff }}$, which represents the ligand field, $D$ denotes the energy difference between the HS state and LS state, and $g$ denotes the ratio of the degeneracies of the HS state and LS state. The order parameter of these models (6) and (8) is defined as

$$
m \equiv \frac{\sum_{i}^{N} \sigma_{i}}{N}
$$

which is related to the fraction of HS molecules, $f_{\mathrm{HS}}$, as $m=2 f_{\mathrm{HS}}-1$.

In order to see the competition between the short-range interaction and the lattice distortion due to the molecular size difference, we consider the model (6) along the coexistent line, $\langle m\rangle=0$, as we studied in the previous work for the Ising model. Thus we set $D-\left(k_{\mathrm{B}} T / 2\right) \log g=0$. For simplicity, this situation is described by the present model (6) in which $D=0$ and $g=1$. In this model, a ferromagnetic second-order phase transition takes place at the critical temperature $T_{\mathrm{c}}$. Below $T_{\mathrm{c}}$, there exist two different ordered states, the HS state and the LS state.

It has been found that in the pure elastic interaction model (8), the spin configuration is uniform at the critical point, with no large-scale clustering. ${ }^{2}$ The phase transition belongs to the mean-field (MF) universality class, and the spin correlation function approaches a non-zero constant in the long-distance limit.

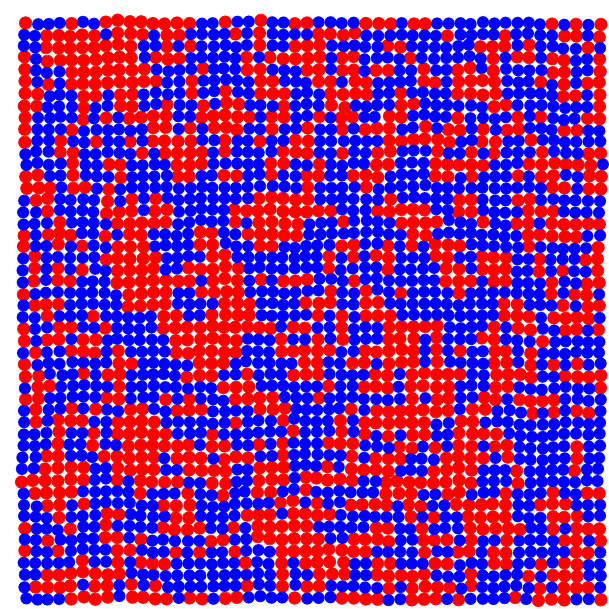

FIG. 1: (Color online) Typical configuration of the mixed elastic and short-range interaction model at the critical point. $R_{\mathrm{H}} / R_{\mathrm{L}}=1.1$, for which $T_{\mathrm{c}}=0.54575$. Light gray (red online) filled circles denote HS state spins, and dark gray (blue online) filled circles denote LS state spins.

The origin of the long-range interaction in the elastic model is lattice distortion due to the size difference between the HS and LS states. We expect that the ratio of the radii $R_{\mathrm{L}} / R_{\mathrm{H}}$ controls the strength of the effective long-range interaction. Namely, $R_{\mathrm{L}} / R_{\mathrm{H}}$ plays a role similar to $\alpha$ in the previous hybrid model (1). The strength of the long-range interaction is given by the elastic energy, which is of the order of $\left(k_{1} / 2\right)\left(R_{\mathrm{H}}-R_{\mathrm{L}}\right)^{2} \propto\left(1-\frac{R_{\mathrm{L}}}{R_{\mathrm{H}}}\right)^{2}$. We therefore consider that the parameter to indicate the strength of the long-range interaction is given by

$$
\alpha \propto\left(1-\frac{R_{\mathrm{L}}}{R_{\mathrm{H}}}\right)^{2} .
$$

With this relation, we expect that the relations (4) and (5) take the following forms,

$$
T_{\mathrm{c}}\left(\frac{R_{\mathrm{L}}}{R_{\mathrm{H}}}\right)-T_{\mathrm{c}}^{\mathrm{IS}} \propto\left(1-\frac{R_{\mathrm{L}}}{R_{\mathrm{H}}}\right)^{\frac{8}{7}},
$$

and

$$
\xi_{\mathrm{c}}\left(\frac{R_{\mathrm{L}}}{R_{\mathrm{H}}}, L\right)=L f\left(L\left(1-\frac{R_{\mathrm{L}}}{R_{\mathrm{H}}}\right)^{\frac{8}{7}}\right)
$$


respectively. As in our previous work, ${ }^{1}$ as long as the value of $1-R_{\mathrm{L}} / R_{\mathrm{H}}$ is small, we expect these formulae to be correct. In Fig. 1, we depict a typical configuration of the elastic and short-range interaction model at its critical temperature. Unlike the pure short-range Ising model, the spin configuration is uniform with no large-scale clustering, even at the critical temperature. A lattice distortion also occurs, and we observe an uneven system surface.

\section{CRITICAL TEMPERATURE}

In this section, we perform MC simulations to test the scaling relation for the critical temperature (11). For the simulation, we adopt the NPT-MC method ${ }^{15}$ for the isothermal-isobaric ensemble with the number of molecules $N$, the pressure of the system $P$, and the temperature $T$. In this paper, in order to exclude other effects than those due to the elastic interaction through distortion, we fix $P=0$. We also fix the spring constants as $k_{1}=40$ and $k_{2}=4$ as in our previous work. ${ }^{2}$ The critical temperature of the pure elastic interaction model $(8)$ is $T_{\mathrm{c}}^{\text {Elastic }} \simeq 0.2$ for $R_{\mathrm{H}}=1.1,{ }^{2}$ and we choose $J=0.1$ in order to keep the two terms in the present model (6) of comparable magnitude near the critical temperature. Therefore, $T_{\mathrm{c}}^{\mathrm{IS}}=0.2269 \cdots$ in these units on the square lattice. We use a standard Metropolis method, adopting periodic boundary conditions. In most cases, we performed eight independent runs of 4,000,000 Monte Carlo steps per spin (MCSS) for the each data with 100,000 MCSS for the initial equilibration. We confirm that the statistical errors are smaller than the marks in the following graphs. We fix $R_{\mathrm{L}}=1.0$ and choose $R_{\mathrm{H}}=1.005,1.008,1.010,1.015,1.02,1.05$, and 1.1 .

We have previously pointed out that even infinitesimally weak long-range interactions become dominant in the thermodynamic limit. ${ }^{1}$ In the case of the previous hybrid model (1), this property is explained by the fact that in a well coarse-grained Hamiltonian, the long-range interactions become stronger than the short-range ones. We need systems sufficiently large that clusters caused by the short-range interactions can be regarded as block spins. ${ }^{1}$ Here we assume that this size dependent crossover phenomenon also takes place in the present model (6).

Here, in order to estimate the critical temperature, we adopt a method we also used in our previous paper. We use the crossing point of the forth-order Binder cumulant ${ }^{17} U_{4}(L)$ for different system sizes to estimate the critical temperature $T_{\mathrm{c}}\left(R_{\mathrm{L}} / R_{\mathrm{H}}\right)$ with high precision. The cumulant is defined as

$$
U_{4}(L) \equiv 1-\frac{\left\langle m^{4}\right\rangle_{L}}{3\left\langle m^{2}\right\rangle_{L}^{2}},
$$

where $m=1 / L^{2} \sum_{i} \sigma_{i}$. In the case of the pure elastic interaction model (8) on the square lattice with periodic boundary conditions, the fixed-point value of the cumulant ${ }^{2}$ is the same as the exact value for the infinite-range interaction model $(3), U_{4}^{\mathrm{MF}} \simeq 0.27 \cdots{ }^{18,19}$ With the radii ratio $R_{\mathrm{L}} / R_{\mathrm{H}}=1$, the present model (6) is equivalent to the pure Ising model on the square lattice with periodic boundary conditions. For this case the fixed-point value of the cumulant is the same as the value for the Ising model $(2), U_{4}^{\mathrm{IS}} \simeq 0.61 \cdots$ on the square lattice. ${ }^{20}$ Other shapes of the system, boundary conditions, and anisotropy may lead to different values of $U_{4}$ at the crossing point. ${ }^{21,22}$ 


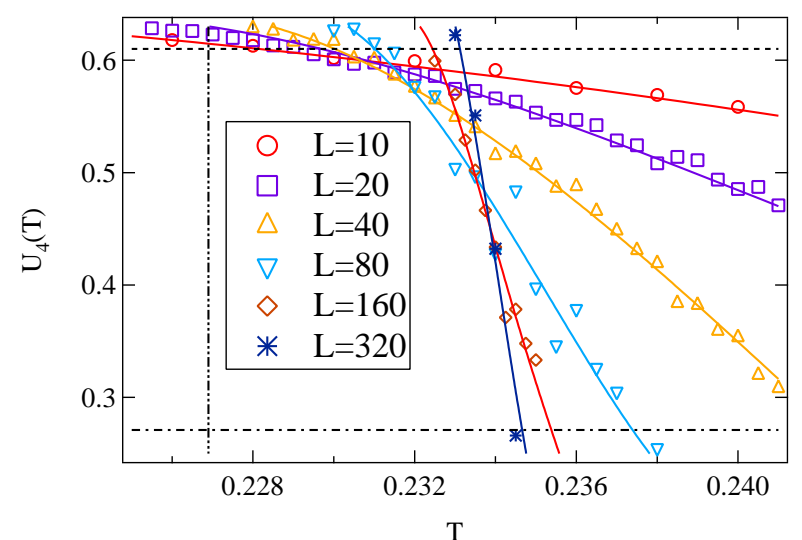

(a)

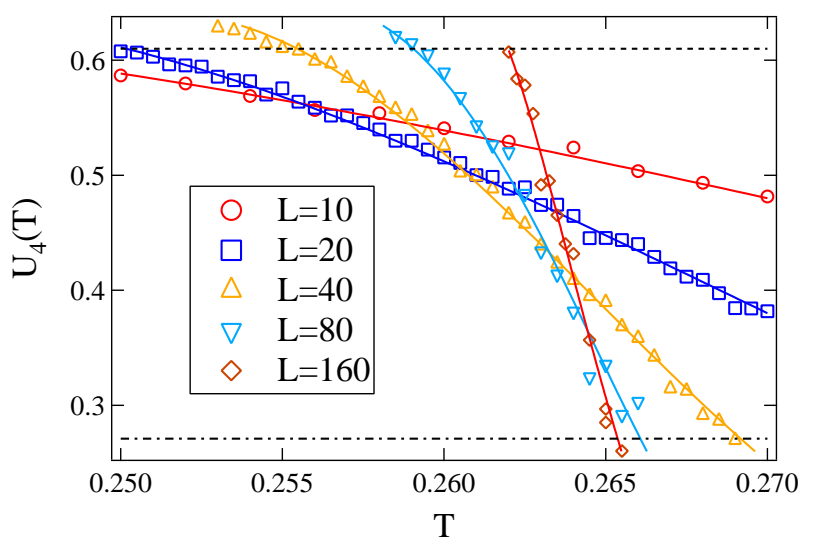

(c)

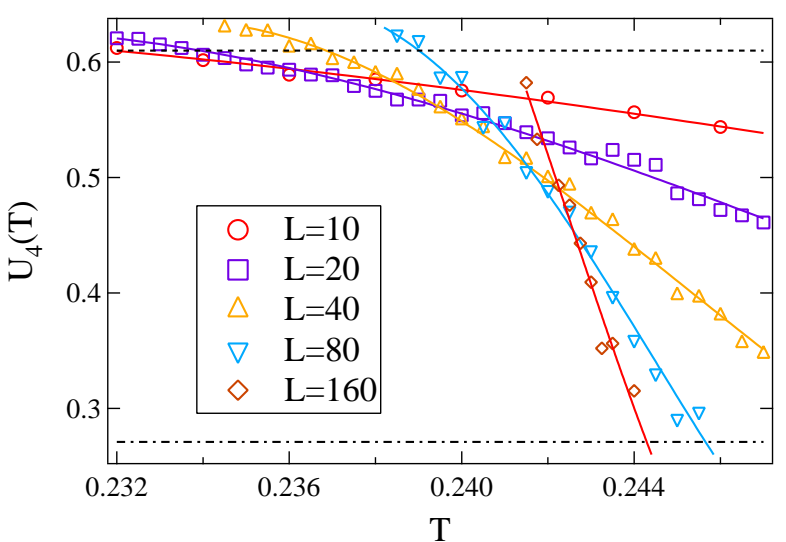

(b)

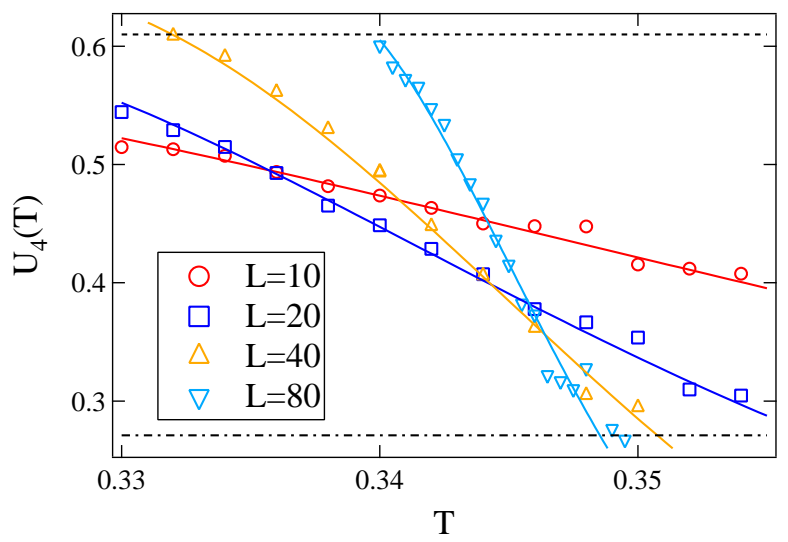

(d)

FIG. 2: (Color online) Temperature dependence of the Binder cumulant $U_{4}(L)$ for (a) $R_{\mathrm{H}}=1.005$, (b) $R_{\mathrm{H}}=1.01$, (c) $R_{\mathrm{H}}=1.02$, and (d) $R_{\mathrm{H}}=1.05$. Points denote Monte Carlo data, and the solid lines are polynomial fits. The upper and lower horizontal lines denote the fixed-point values for the Ising model $\left(U_{4}^{\mathrm{IS}} \simeq 0.61\right)$ and the infinite-range model $\left(U_{4}^{\mathrm{MF}} \simeq 0.27\right)$, respectively. The left vertical line in (a) represents the critical temperature of the pure Ising model. The linear system size $L$ is $10,20,40,80,160$, and 320 for circles, squares, upward triangles, downward triangles, diamonds, and asterisks, respectively.

In Fig. 2, we see that the crossing points of the Binder cumulant decrease toward the mean-field fixed-point value, $U_{4}^{\mathrm{MF}} \simeq 0.27 \ldots 18,19$ from the Ising fixed-point value, $U_{4}^{\mathrm{IS}} \simeq 0.61 \ldots 20$ as $L$ increases. This indicates that a size dependent crossover occurs, and that the critical point of this model belongs to the mean-field universality class. We estimate the critical temperature as follows. Assuming that the critical behavior of the model belongs to the mean-field universality class, we get a series of upper bounds on the critical temperature as the temperature at which $U_{4}(L)$ crosses $U_{4}^{\mathrm{MF}}$. Lower bounds are given by the cumulant-crossing temperatures of $U_{4}(L)$ and $U_{4}(L / 2)$. In Fig. 3, bars denote those upper bounds and lower bounds of the critical temperature, and we plot the middle points of those bounds by bullets. Increasing $L$, the temperature range between the upper bounds and the lower bounds becomes narrow. For each value of $R_{\mathrm{L}} / R_{\mathrm{H}}$, we extrapolate those middle points for the two largest system sizes versus $1 / L$ to obtain the corresponding critical temperature in the thermodynamic limit. With this method, we expect that a critical temperature of a hybrid system with both short-range and long-range interactions can be obtained accurately in general. The data collapse well onto a straight line as shown in Fig. 4, confirming the scaling form (11) for $R_{\mathrm{H}}$ smaller than 1.02. The points fall above the line for large values of $1-R_{\mathrm{L}} / R_{\mathrm{H}}$. In this model, we fix the strength of the Ising interactions, while in the previous hybrid model (1), the factor $1-\alpha$ multiplying the Ising Hamiltonian causes a deviation of the critical temperature in the opposite direction (see Fig. 4 of Ref. 1). 


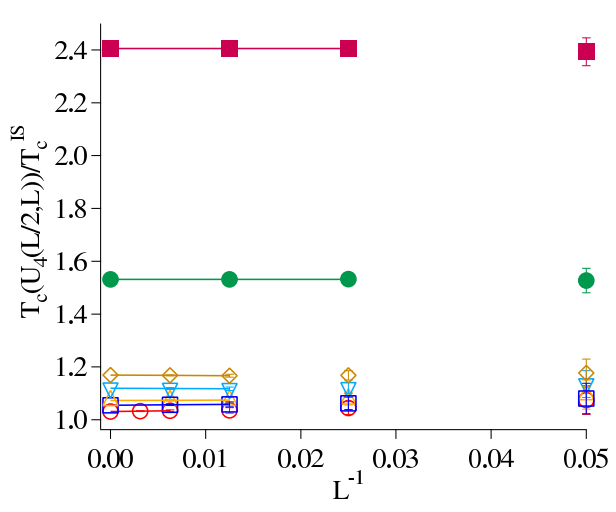

(a)

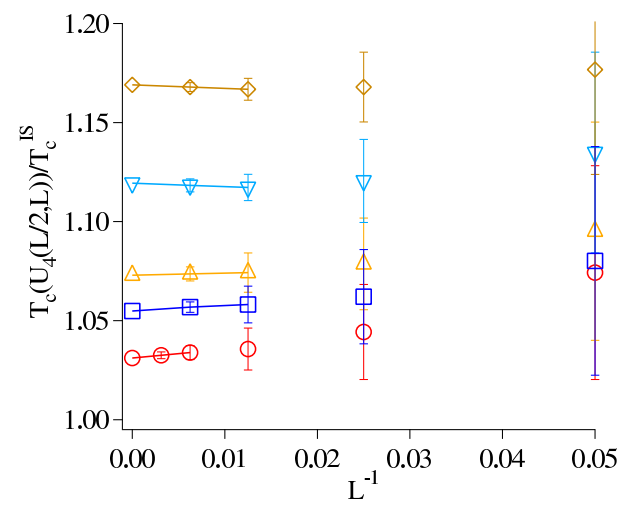

(b)

FIG. 3: (Color online) Size dependence of the crossing point of the Binder cumulant (a) and an enlarged view of the same for small $R_{\mathrm{H}}$ (b). From above to below, we show simulation results with $L=20,40$, and 80 for $R_{\mathrm{H}}=1.10$ and $1.05, L=20,40,80$, and 160 for $R_{\mathrm{H}}=1.02,1.015,1.01$ and 1.008, $L=20,40,80,160$ and 320 for $R_{\mathrm{H}}=1.005$. The points at $1 / L=0$ are linear extrapolations from the two smallest nonzero values of $1 / L$. Filled squares, filled circles, diamonds, downward triangles, upward triangles, open squares, and open circles represent $T_{\mathrm{c}}(L)$ for $R_{\mathrm{H}}=1.10, R_{\mathrm{H}}=1.05, R_{\mathrm{H}}=1.02, R_{\mathrm{H}}=1.015, R_{\mathrm{H}}=1.010$, $R_{\mathrm{H}}=1.008$, and $R_{\mathrm{L}}=1.005$, respectively.

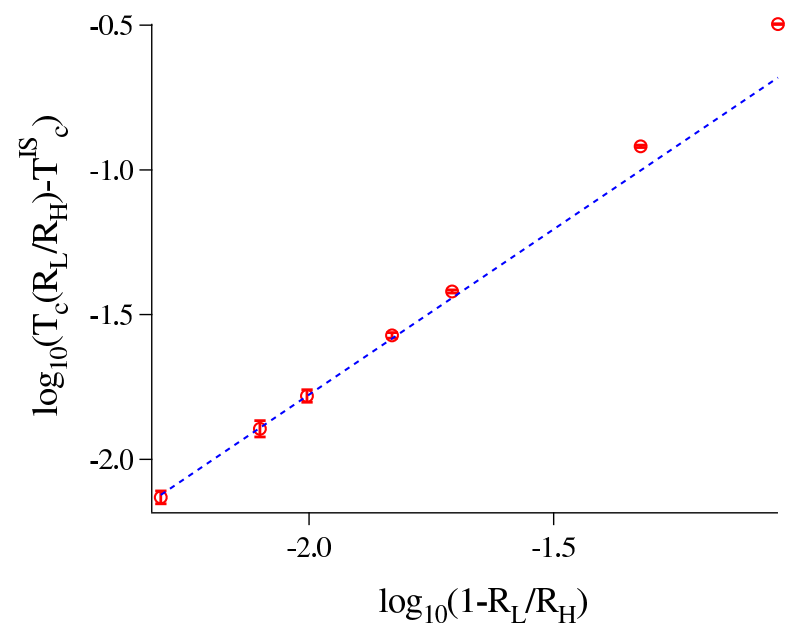

FIG. 4: (Color online) The $\left(1-\frac{R_{\mathrm{L}}}{R_{\mathrm{H}}}\right)$ dependence of the shift of the critical temperature in a log-log plot. The oblique dashed line (blue online) represents a numerical fit of the 4 leftmost points to $\left(1-\frac{R_{\mathrm{L}}}{R_{\mathrm{H}}}\right)^{\frac{8}{7}}$.

\section{CORRELATION LENGTH}

In this section, we perform MC simulations to test the scaling relation for the correlation length at the critical point (12). We previously calculated the critical correlation length in the previous hybrid model (1) from MC simulations. ${ }^{1}$ In that paper, we used the spin-correlation function $c(r)=\left\langle\sigma\left(r^{\prime}\right) \sigma\left(r^{\prime}+r\right)\right\rangle$ to estimate the correlation length, excluding the contribution of long-range correlations from the correlation function. In the present model (6), because of strong anisotropy of the correlations, ${ }^{2}$ it is not practical to calculate the correlation length $\xi\left(R_{\mathrm{L}} / R_{\mathrm{H}}\right)$ with this method.

Here we instead obtain the correlation length from the structure factor,

$$
S(\boldsymbol{k})=\frac{1}{N} \sum_{l, m}\left\langle\sigma_{l} \sigma_{m}\right\rangle e^{i \boldsymbol{k} \cdot \boldsymbol{r}_{l, m}}
$$

which is readily measured in scattering experiments. All the contributions of the long-range correlations are given 
by $S(\boldsymbol{k}=0)$, so we can easily exclude them from the calculation. Here we note that there exist many experimental studies on the structure factors of magnetic materials, ${ }^{29}$ obtained by neutron scattering. In the case of spin-crossover materials, the structures have been studied by single-crystal x-ray diffraction experiments. ${ }^{30,31}$ The structure factor for the HS/LS state domains discussed in the present paper can be obtained from diffuse x-ray scattering.

In the pure short-range Ising model at the critical temperature, the structure factor has its peak at $\boldsymbol{k}=0$ with infinitesimally narrow width in the thermodynamic limit. However, in the hybrid model with both short-range and long-range interactions, the system prefers a spatially uniform configuration and the cluster size is suppressed even at the critical temperature, and the peak has a finite width in the thermodynamic limit. Here we obtain the correlation length at the critical point, $\xi_{c}$ by calculating the characteristic peak width of the structure factor.

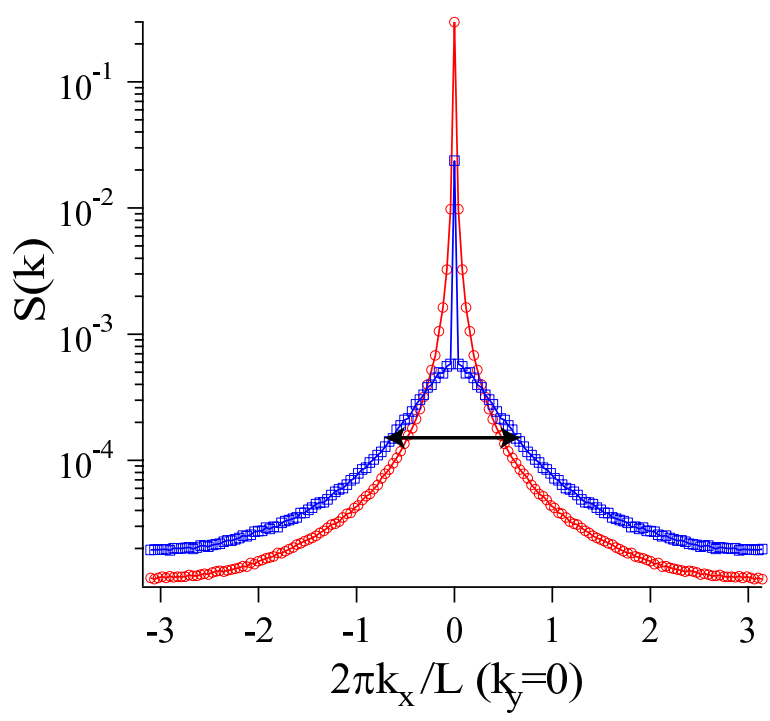

(a)

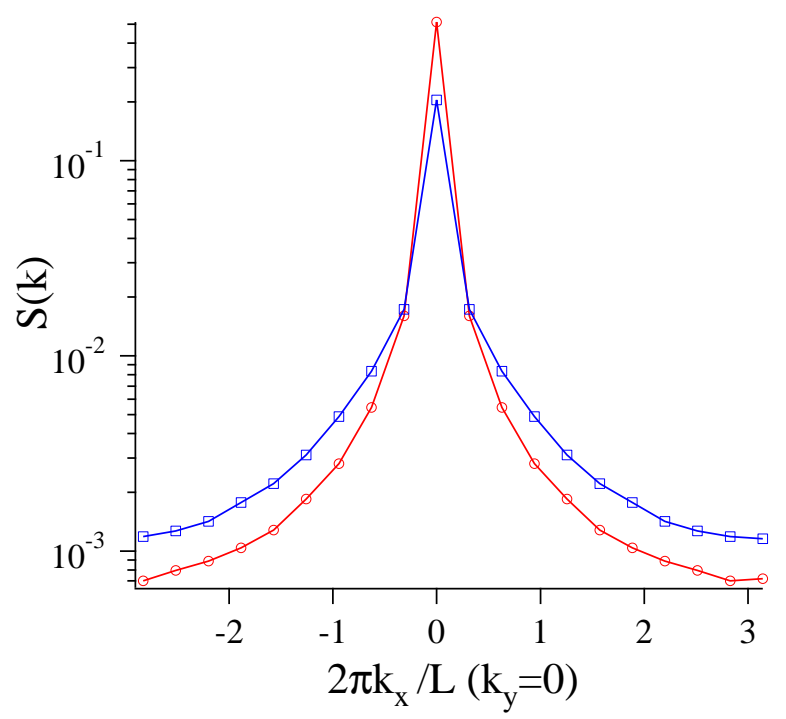

(b)

FIG. 5: (Color online) The structure factor of the pure short-range Ising model (circles) and the hybrid model with $R_{\mathrm{H}}=1.02$ (squares), at each its critical point. (a) $L=160$ and (b) $L=20$.

In Fig. 5, we depict the structure factor of the pure Ising model at its critical temperature (circles) together with that of the hybrid model at its critical temperature (squares). Figure 5 (a) shows the structure factor of the system for $L=160$, and Fig. 5 (b) is for $L=20$. We find a qualitative difference between these figures. Namely, we find a flat region in $S(\boldsymbol{k})$ of the hybrid model around $\boldsymbol{k}=\mathbf{0}$ in Fig. 5 (a), while $S(\boldsymbol{k})$ for both the pure short-range Ising model and the hybrid model show similar peaks in Fig. 5 (b).

The structure factor of the hybrid model consists of two parts: A $\delta$-function at $\boldsymbol{k}=\mathbf{0}$ and a broad peak reflecting short-range order with the correlation length $\xi_{\mathrm{c}}$. The two-parts are superimposed in Fig. 5 (a). The flat region belongs to the diffuse peak, which is due to the finite cluster structure of the hybrid model. We find that for sufficiently large systems, the diffuse peak and the $\delta$-peak at $\boldsymbol{k}=\mathbf{0}$ are well distinguished. In those systems, we define the characteristic peak width $\left|\boldsymbol{k}_{\text {peak }}\right|$ as the spectral peak width of the diffuse peak as shown by the arrows in Fig. 5 (a).

In Fig. 5 (b), for small $L$, the resolution in $\boldsymbol{k}$-space $(2 \pi / L)$ is rather coarse, so the data points do not reflect the finite width of the peak in the hybrid model well. It is hard to distinguish between the structure factors of the Ising model and the hybrid model in such small systems.

We note that the sum rule of the structure factor is $\sum_{\boldsymbol{k}} S(\boldsymbol{k})=N$. In the hybrid model, $S(\boldsymbol{k})$ for large $|\boldsymbol{k}|$ is larger than that of the pure short-range Ising model because $S(\boldsymbol{k})$ around $\boldsymbol{k}=\mathbf{0}$ is suppressed in the hybrid model.

We can estimate the correlation length $\xi$ from the structure factor by considering the first moment of $k^{-1}$,

$$
\left\langle k^{-1}\right\rangle \equiv \sum_{k \neq 0} \frac{1}{|\boldsymbol{k}|} S(\boldsymbol{k}),
$$

where $k=|\boldsymbol{k}|$. In our present model, the correlation function of the spin configuration is given by ${ }^{2,23}$

$$
\left\langle\sigma_{i} \sigma_{j}\right\rangle=c\left(\boldsymbol{r}_{i, j}\right)=c^{\mathrm{SR}}\left(\left|\boldsymbol{r}_{i}-\boldsymbol{r}_{j}\right|\right)+c^{\mathrm{LR}} .
$$


Here, $c^{\mathrm{SR}}\left(\left|\boldsymbol{r}_{i}-\boldsymbol{r}_{j}\right|\right)$ denotes the contributions from the short-range interactions, and $c^{\mathrm{LR}}$ denotes those from the long-range interactions. We note that the long-range correlation $c^{\mathrm{LR}}$ does not depend on the distance $\left|\boldsymbol{r}_{i}-\boldsymbol{r}_{j}\right|$. In Eq. (15), the contribution from the long-range correlations $c^{\mathrm{LR}}$ (i.e., $S(\boldsymbol{k}=\mathbf{0})$ ) is excluded, so we use the asymptotic formula for the pure short-range Ising model, ${ }^{24}$

$$
c(r) \propto \frac{1}{r^{2-d+\eta}} e^{-r / \xi}
$$

In the thermodynamic limit, we replace the sum in (15) by an integral:

$$
\begin{aligned}
\left\langle k^{-1}\right\rangle & =\int_{k=2 \pi / L}^{\infty} \int_{\phi=0}^{2 \pi} k d k d \phi \frac{1}{k} \int_{r=0}^{\infty} \int_{\theta=0}^{2 \pi} r d r d \theta e^{i k r \cos \theta} c(r) \\
& \propto \int_{k=0}^{\infty} d k \int_{r=0}^{\infty} d r r^{1-\eta} e^{-r / \xi} J_{0}(k r) \\
& =\xi^{1-\eta} \int_{K=0}^{\infty} \int_{R=0}^{\infty} R^{-\eta} e^{-R} J_{0}(K) d K d R \\
& \propto \xi^{1-\eta}=\xi^{3 / 4} .
\end{aligned}
$$

In the last line, we use the substitutions, $K=k r, R=r / \xi$, and $J_{0}(K)$ is the Bessel function. There is no singularity in the integral, and $\left\langle k^{-1}\right\rangle$ gives a power of the correlation length. Therefore we define the critical correlation length $\xi_{\mathrm{c}}$ at the critical temperature $T_{\mathrm{c}}$ as follows,

$$
\xi_{\mathrm{c}} \propto\left\langle k^{-1}\right\rangle_{\mathrm{c}}^{\frac{4}{3}}
$$

where $\left\langle k^{-1}\right\rangle_{\mathrm{c}}$ represents the value at the critical point. The scaling relation for $\left\langle k^{-1}\right\rangle_{\mathrm{c}}$ is, by making use of $(12)$,

$$
\left\langle k^{-1}\right\rangle_{\mathrm{c}}^{\frac{4}{3}} \propto \xi_{\mathrm{c}}=L f\left(L\left(1-\frac{R_{\mathrm{L}}}{R_{\mathrm{H}}}\right)^{\frac{8}{7}}\right)
$$

where $g(x) \propto \frac{1}{x}$ for $x \rightarrow \infty$.

We obtained $\left\langle k^{-1}\right\rangle_{\mathrm{c}}$ and the corresponding values of $\xi_{\mathrm{c}}$ for various values of $L$ and $R_{\mathrm{L}} / R_{\mathrm{H}}$. Those are plotted in Fig. 6. In the enlarged view of Fig. 6 for small $L$, we find inflection points. For small $L, \xi_{\mathrm{c}}\left(R_{\mathrm{L}} / R_{\mathrm{H}}, L\right)$ grows faster in the hybrid model than in the pure Ising model.

Here let us consider the reason for this behavior. This fast growth is due to the coarse resolution in $\boldsymbol{k}$-space. The characteristic peak width of the hybrid model does not represent well the structure factor in the small systems (Fig. 5(b)), and the correlation length of the hybrid model is overestimated in Eq. (23). This extra size dependence for small size comes from the discontinuous nature of $\boldsymbol{k}$-space, and is not essential for the present purpose.

For large $L$, the growth of $\xi_{\mathrm{c}}\left(R_{\mathrm{L}} / R_{\mathrm{H}}, L\right)$ becomes much slower than in the pure Ising model. For $R_{\mathrm{H}}=1.02$ and $L=320, \xi_{\mathrm{c}}\left(R_{\mathrm{L}} / R_{\mathrm{H}}, L\right)$ is almost saturated. For other values of $R_{\mathrm{H}}, \xi_{\mathrm{c}}\left(R_{\mathrm{L}} / R_{\mathrm{H}}, L\right)$ is expected to saturate for still larger systems. It means that for sufficiently large systems, the difference of the structure factor of the hybrid model and the pure short-range model is clear. 


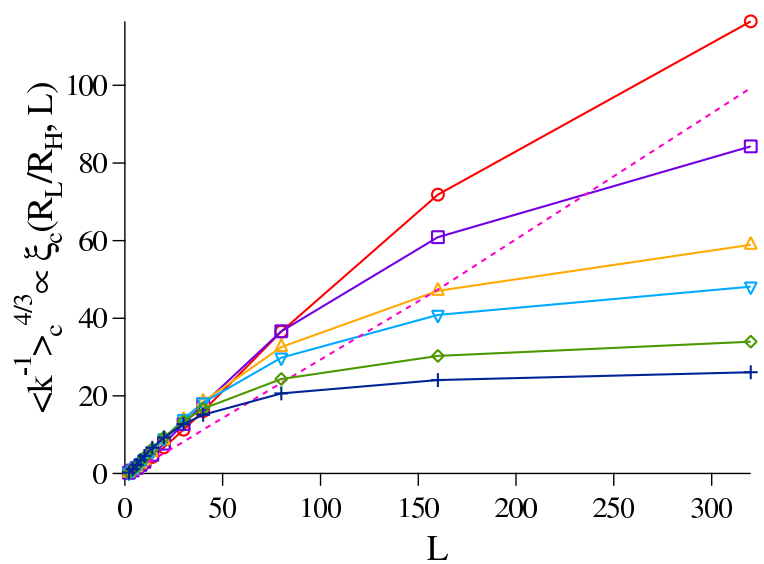

(a)

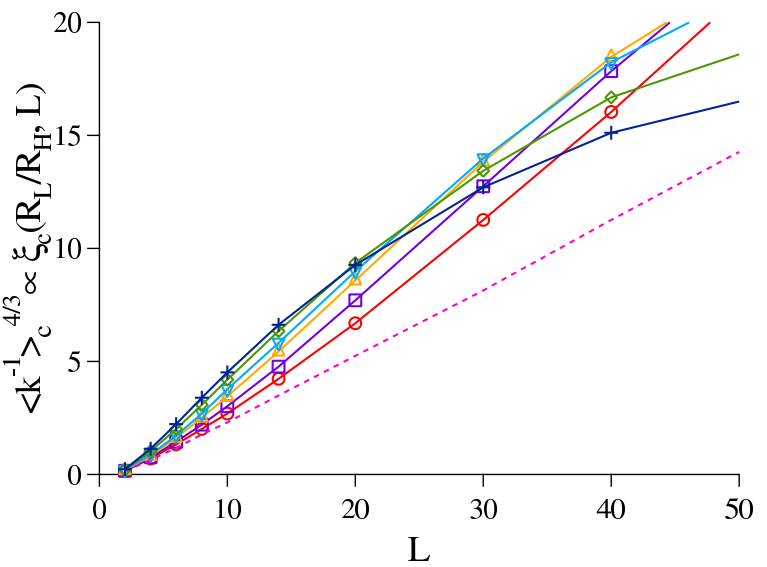

(b)

FIG. 6: (Color online) The size dependence of $\left\langle k^{-1}\right\rangle_{\mathrm{c}}^{\frac{4}{3}} \propto \xi_{\mathrm{c}}$ at the critical point $T_{\mathrm{c}}\left(R_{\mathrm{L}} / R_{\mathrm{H}}\right)$ (a), and an enlarged view of the same for small $L$ (b). $L=2,4,6,8,10,14,20,30,40,80,160$ and 320 . Circles, squares, upward triangles, downward triangles, diamonds and crosses represent $T=0.23107$ for $R_{\mathrm{H}}=1.003, T=0.23428$ for $R_{\mathrm{H}}=1.005, T=0.23965$ for $R_{\mathrm{H}}=1.008$, $T=0.24345$ for $R_{\mathrm{H}}=1.01, T=0.2537$ for $R_{\mathrm{H}}=1.015$ and $T=0.2649$ for $R_{\mathrm{L}}=1.02$, respectively. The dashed line (blue online) represents $T=0.2269$ for $R_{\mathrm{H}}=1.00$, the pure Ising-model limit.

The same behavior is found in the previous hybrid model (1). In both models, the previous hybrid model (1) and the present model (6), the long-range interactions suppress the clustering of spins. Thus there appear tightly correlated effective block spins. In the coarse-grained Hamiltonian with the block spins, the long-range interactions become effectively stronger than the short-range interactions. ${ }^{1}$

According to this picture, for $L$ smaller than the clusters caused by the short-range interaction, the long-range interaction is irrelevant. Thus, for small $L$, the system is an effective short-range interacting system. On the other hand, for sufficiently large $L$, the system is an effective long-range interacting system. We refer the crossover length as $L_{\mathrm{cl}}$, which we define as $L_{\mathrm{cl}}=1 /\left|\boldsymbol{k}_{\text {peak }}\right|$. The inflection points in Fig. 6 also indicate the crossover length of the system size, $L_{\mathrm{cl}}$. For $L>L_{\mathrm{cl}}$, the correlation length at the critical point, $\xi_{\mathrm{c}}\left(R_{\mathrm{L}} / R_{\mathrm{H}}, L\right)$, tends to be saturated. For $L<L_{\mathrm{cl}}, \xi_{\mathrm{c}}\left(R_{\mathrm{L}} / R_{\mathrm{H}}, L\right)$ grows faster than linear.

We also found that the peak position of $\tilde{\chi}(L) \equiv \frac{1}{N}\left(\left\langle M^{2}\right\rangle-\langle|M|\rangle^{2}\right)$ shows similar behavior. The peak position, the effective 'critical point' for a system of size $L$, saturates at the critical temperature in the thermodynamic limit:

$$
T_{c}\left(\frac{R_{\mathrm{L}}}{R_{\mathrm{H}}}, L\right) \rightarrow T_{\mathrm{c}}\left(\frac{R_{\mathrm{L}}}{R_{\mathrm{H}}}, \infty\right) .
$$

As in the previous work, ${ }^{1}$ we found a non-monotonic dependence of the peak position as a function of $L$. For small $L$, the peak positions approach $T_{\mathrm{c}}$ from the high-temperature-side, while for large $L$, the peak position is on the low-temperature-side and eventually approaches $T_{\mathrm{c}}$ from below. This non-monotonic behavior indicates the crossover from the effective short-range system to the effective long-range system. It is an interesting problem to study the relation between the crossover phenomena in $\tilde{\chi}(L)$ and the correlation length $\xi_{\mathrm{c}}$ in Fig. 6 . We expect that this general system size dependence occurs in any hybrid model where effective spins play an important role.

In appendix A, we discuss in detail how the correlation lengths are measured. In Fig 7, we plot the data for $L>L_{\mathrm{cl}}\left(R_{\mathrm{L}} / R_{\mathrm{H}}\right)$ in a scaling plot of the form (23). We find that the data collapse onto a scaling function and thus we conclude that (23) is justified. 


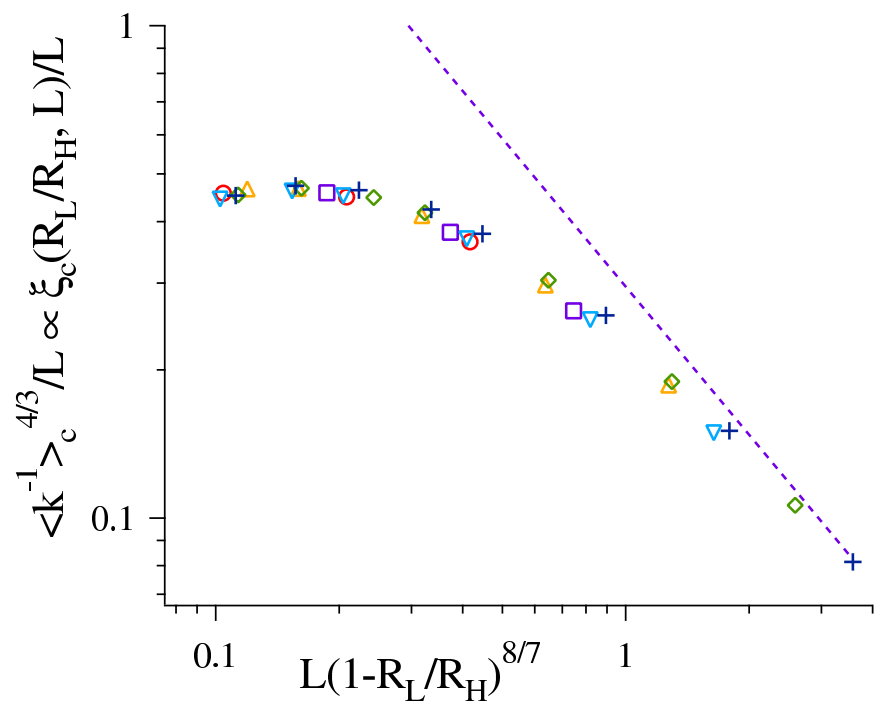

FIG. 7: (Color online) Scaling plot of the correlation length at the critical point. Circles, squares, upward triangles, downward triangles, diamonds, and crosses represent $T=0.23107$ for $R_{\mathrm{H}}=1.003, T=0.23428$ for $R_{\mathrm{H}}=1.005, T=0.23965$ for $R_{\mathrm{H}}=1.008, T=0.24345$ for $R_{\mathrm{H}}=1.01, T=0.2537$ for $R_{\mathrm{H}}=1.015$, and $T=0.2649$ for $R_{\mathrm{L}}=1.02$, respectively. Data are included only for $L>L_{\mathrm{cl}}$. The dashed line is proportional to $y=x^{-1}$. The data are in good agreement with the scaling relation, but they converge more gradually to the asymptotic formula than when the correlation length is calculated from the correlation function. ${ }^{1}$

\section{SUMMARY AND FURTHER DISCUSSION}

In general, spin-crossover and related materials have both short-range and long-range interactions. If the elastic potential is chosen spin-state dependent ${ }^{27,28}$, in which different potential functions (coupling constants) are given for LS-LS, LS-HS, and HS-HS molecular pairs, it is considered that the interaction has intrinsically both short-range and long-range components. In this paper, we have studied critical properties of the present model (6) with elastic and short-range interactions. By Monte Carlo simulations we confirmed that the present model obeys a scaling relation for the shift of the critical temperature as a function of the strength of the long-range interaction, which is determined by the ratio of the radii of the molecules in both states. We similarly confirmed that the present model also obeys a scaling relation for the correlation length at the critical point, which was found in our previous work for the hybrid model with infinite-range and nearest-neighbor interactions. ${ }^{1}$ Although the origin of the long-range interaction is very different in the previous model and the present model, we found several kinds of universality in their critical properties.

In the present model, because of the anisotropy in the correlation function, we estimated the correlation length from the structure factor. By this method, the correlation length can be measured in scattering experiments on real materials. Because spin-crossover materials usually undergo first-order phase transitions, experiments on the structure factors of these materials have been so far only done for HS/LS ordered phase. ${ }^{30,31}$ Our results show that the structure factor of spin-crossover materials should exhibit a peak with finite width at the critical point due to the elastic interaction. We hope such unique behavior will be observed by single crystal x-ray diffraction along the coexistence line and at the critical point.

It was confirmed that the present model (6) possesses an effective long-range interaction. The details of the effective long-range interactions introduced by the elastic degrees of freedom in the present model are not known. Only for $d=1,{ }^{25}$ it has been shown rigorously that the model can be mapped onto an short-range Ising model. There has also been much previous research on three-dimensional elastic solids, and it is generally argued that the dominant long-range interactions are of a dipole-dipole nature, $\sim 1 / r^{3} .{ }^{26}$ Although the effects of distortions in the present model are not identical to those in the classical elastic media for which these results were obtained, we assume that the elastically mediated interactions in our model also are of such long-range type. However, in our present model the spin correlation function shows infinite-range correlations above the critical temperature. ${ }^{2,23}$ Therefore, we assume that there are similar but unknown infinite-range interactions in the present model. Understanding the mechanism by which the infinite-range interactions arise remains an intriguing problem for future research. 


\section{Acknowledgments}

The present work was partially supported by the Mitsubishi Foundation, Grant-in-Aid for Scientific Research on Priority Areas, KAKENHI (C) 23540381, and also the Next Generation Super Computer Project, Nanoscience Program from MEXT of Japan. T.M. acknowledges the support from JSPS (Grant No. 227835). The numerical calculations were supported by the supercomputer center of ISSP of University of Tokyo. Work at Florida State University was supported in part by U.S. National Science Foundation Grants No. DMR-0802288 and DMR-1104829.

\section{Appendix A: Calculating the correlation length $\xi$}

In the previous work on the model defined in (1), there exist both short-range and infinite-range interactions, but no anisotropy. Therefore we could exclude the contribution of the long-range correlations uniformly from the correlation function, obtaining the correlation length from the following relation,

$$
\xi=\frac{\int_{0}^{L / 2}(c(r)-c(L / 2)) r d r}{\int_{0}^{L / 2}(c(r)-c(L / 2)) d r} .
$$

Here, $c(L / 2)$ which is proportional to $\frac{1}{\sqrt{N}}$ is a good approximation for the large- $r$ limit of $c(r)$.

In the present paper, we adopt another method because of the strong anisotropy in the correlation function. In a $d$-dimensional system, we can estimate $\xi$ from

$$
\left\langle k^{1-d}\right\rangle \equiv \sum_{k \neq 0} \frac{1}{|\boldsymbol{k}|^{d-1}} S(\boldsymbol{k}) \propto \xi^{2 d-3-\eta} .
$$

In the present model defined by (6) with very weak elastic interactions, there exist rather large, but finite clusters at the critical point due to the short-range interactions. In (A2), we sum the structure factor with importance $1 /|\boldsymbol{k}|^{d-1}$ in order to avoid divergences and to collect the contributions from length scales on the order of $L$, using the asymptotic form of the correlation function (17).

1 T. Nakada, P. A. Rikvold, T. Mori, M. Nishino, and S. Miyashita, Phys. Rev. B 84, 054433 (2011).

2 S. Miyashita, Y. Konishi, M. Nishino, H. Tokoro, and P. A. Rikvold, Phys. Rev. B 77, 014105 (2008).

${ }^{3}$ K. L. Ronayne, H. Paulsen, A. Höfer, A. C. Dennis, J. A. Wolny, A. I. Chumakov, V. Schünemann, H. Winkler, H. Spiering, A. Bousseksou, P. Gütlich, A. X. Trautwein, and J. J. McGarvey, Phy. Chem. Chem. Phys. 84685 (2006).

${ }^{4}$ P. Gütlich, A. Hauser, and H. Spiering, Angew. Chem., Int. Ed. Engl. 33, 2024(1994), and references therein.

5 J.A. Real, H. Bolvin, A. Bousseksou, A. Dworkin, O. Kahn, F. Varret, and J. Zarembowitch, J. Am. Chem. Soc. 114, 4650 (1992).

6 O. Kahn and C. J. Martinez, Science 279, 44 (1998).

7 J. F. Létard, P. Guionneau, L. Rabardel, J. A. K. Howard, A. E. Goeta, D. Chasseau, and O. Kahn, Inorg. Chem. 37, 4432 (1998).

8 A. Hauser, J. Jeftić, H. Romstedt, R. Hinek, and H. Spiering, Coord. Chem. Rev. 190-192, 471 (1999).

9 N. Shimamoto, S. Ohkoshi, O. Sato, and K. Hashimoto, Inorg. Chem. 41, 678 (2002).

10 G. Molnár, A. Bousseksou, A. Zwick, J. J. McGarvey, Chem. Phys. Lett. 367, 593 (2003).

11 M. Nishino, K. Boukheddaden, Y. Konishi, and S. Miyashita, Phys. Rev. Lett. 98, 247203 (2007).

12 C. Enachescu, L. Stoleriu, A. Stancu, and A. Hauser, Phys. Rev. Lett. 102, 257204 (2009).

13 M. Nishino, C. Enachescu, S. Miyashita, K. Boukheddaden, and F. Varret, Phys. Rev. B 82, R020409 (2010).

14 Y. Konishi, H. Tokoro, M. Nishino, and S. Miyashita, Phys. Rev. Lett. 100, 067206 (2008).

15 I. R. McDonald, Chem. Phys. Lett. 3, 241 (1969).

16 N. Huby, L. Guérin, E. Collet, L. Toupet, J. C. Ameline, and H. Cailleau, T. Roisnel, T. Tayagaki, and K. Tanaka, Phys. Rev. B 69, 020101(R) (2004).

17 K. Binder, Phys. Rev. Lett. 47, 693 (1981).

18 E. Brezin and J. Zinn-Justin, Nucl. Phys. B 257, 867 (1985).

19 E. Luijten and H. W. J. Blöte, Int. J. Mod. Phys. C 6, 359 (1995).

20 G. Kamieniarz and H. W. J. Blöte, J. Phys. A 26, 201 (1993).

21 X. S. Chen and V. Dohm, Phys. Rev. E 70, 056136 (2004).

${ }^{22}$ W. Selke and L. N. Shchur, J. Phys. A: Math. Gen. 38, L739 (2005).

${ }^{23}$ Y. Konishi, Graduate thesis, Graduate School of Science, The University of Tokyo, Japan (2007). 
${ }^{24}$ T. T. Wu, B. M. McCoy, C. A. Tracy, and E. Barouch, Phys. Rev. B 13, 316 (1976).

${ }^{25}$ K. Boukheddaden, S. Miyashita, and M. Nishino, Phys. Rev. B 75, 094112 (2007).

26 J. Stewart, O. Pohland and J. M. Gibson, Phys. Rev. B 49, 13848 (1994).

27 W. Nicolazzi, S. Pillet, and C. Lecomte, Phys. Rev. B 78, 174401 (2008).

${ }^{28}$ W. Nicolazzi, S. Pillet, and C. Lecomte, Phys. Rev. B 80, 132102 (2009).

29 H. Ikeda and K. Hirakawa, Solid State Commun. 14, 529 (1974).

${ }^{30}$ P. Guionneau, F. Le. Gac, S. Lakhoufi, A. Kaiba, D. Chasseau, J.-F. Látard, P. Négrier, D. Mondieig, J. A. K. Howard, and J.-M. Láger, J. Phys. Condens. Matter 19326211 (2007).

31 S. Lakhloufi, P. Guionneau, M. H. Lemáe-Cailleau, P. Rosa, and J.-F. Látard, Phys. Rev. B 82, 132104 (2010). 